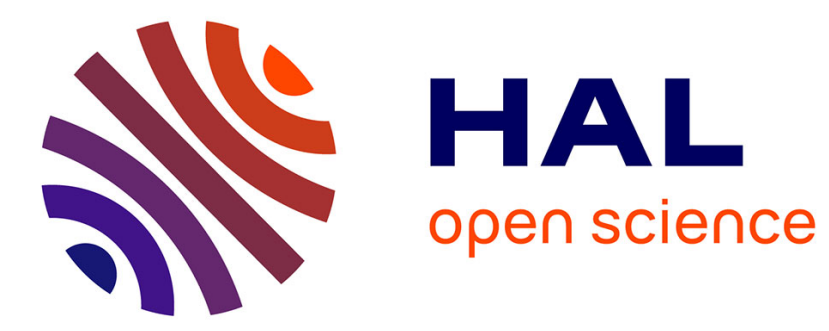

\title{
Multiscale signal processing : isotropic random fields on homogeneous trees
}

\author{
Bernhard Claus, Ghislaine Chartier
}

\section{To cite this version:}

Bernhard Claus, Ghislaine Chartier. Multiscale signal processing : isotropic random fields on homogeneous trees. [Research Report] RR-1709, INRIA. 1992. inria-00076946

\section{HAL Id: inria-00076946 \\ https://hal.inria.fr/inria-00076946}

Submitted on 29 May 2006

HAL is a multi-disciplinary open access archive for the deposit and dissemination of scientific research documents, whether they are published or not. The documents may come from teaching and research institutions in France or abroad, or from public or private research centers.
L'archive ouverte pluridisciplinaire HAL, est destinée au dépôt et à la diffusion de documents scientifiques de niveau recherche, publiés ou non, émanant des établissements d'enseignement et de recherche français ou étrangers, des laboratoires publics ou privés. 


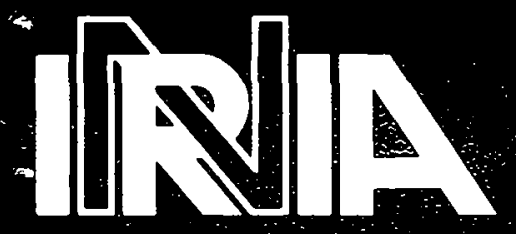

UNITÉ DE RECHERCHE INRIA-RENNES

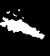

Institut National de Recherche en Informatique et en Automatique

$-$

Domaine de Voluceau

Rocquencourt B.P105

3858 Le Chesnay Cedex France

Tél.:(1)39635511

\section{Rapports de Recherche}

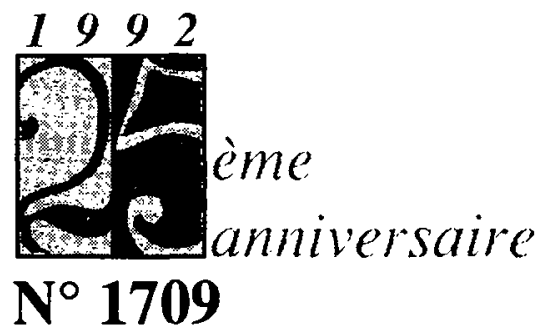

Programme 5

Traitement du Signal, Automatique et Productique

MULTISCALE SIGNAL PROCESSING :

ISOTROPIC RANDOM FIELDS ON HOMOGENEOUS TREES

\author{
Bernhard CLAUS
}

Ghislaine CHARTIER

Juin 1992 


\title{
IRISA
}

INSTITUT DE RECHERCHE EN INFORMATIQUE

ET SYSTEMES ALEATOIRES

Campus Universitaire de Beaulieu 35042 - RENNES CEDEX FRANCE

Tél. : 99847100 - Télex : UNIRISA $950473 \mathrm{~F}$

Télècopie : 99383832

\section{Multiscale Signal Processing: \\ Isotropic Random Fields on Homogeneous Trees}

Publication Interne $n^{\circ} 658$ - Mai 1992 - 28 Pages

Bernhard Claus, Ghislaine Chartier

IRISA/INRIA, Programme 5

\begin{abstract}
In this paper we consider isotropic processes on a homogeneous tree of order $q$, i.e. stochastic processes which covariance function depends only on the distance on the tree. We develop a characterization of isotropic processes via a reflection coefficient sequence. The relation between the covariance sequence and the reflection coefficient sequence of a process is derived by generalized Levinson and Schur recursions respectively. Although these results are easily obtained using an isometric (i.e. non-oriented) approach, the Levinson and Schur recursions are also given from the pyramidal point of view. They turn out to be useful in this form for the implementation of generating and whitening filters $[1,2]$.
\end{abstract}

\section{Traitement du signal multirésolution: champs aléatoires isotropes sur l'arbre homogène}

\section{Résumé}

On considère des processus isotropes sur l'arbre homogène d'ordre q, i.e. des processus dont la fonction de covariance ne dépend que de la distance sur l'arbre. On développe une caractérisation de ces processus isotropes via une suite de coefficients de réflexion. La relation entre la suite de colariances et la suite de coefficients de réflexion est établi par des récurrences de Levinson et Schur généralisées. Ces résultats sont obtenu d'une manière évidente en utilisant une approche isométrique (i.e. non-orienté). On établit les récurrences de Levinson et Schur aussi en utilisant le point de vue pyramidal (i.e. orienté), ce qui donne des résultats intéressants pour l'implémentation de filtres générateurs ou de filtres blanchisseurs $[1,2]$. 


\title{
Multiscale Signal Processing: Isotropic Random Fields on Homogeneous Trees
}

\author{
B.Claus, G.Chartier *
}

May 14, 1992

\section{Introduction}

Recent advances in both mutirate filtering [7] and orthonormal wavelet theory [8] have emphasized the significance of decimation in multiresolution signal or image processing. As discussed in particular in [6] handling successively decimated versions of a given signal amounts to indexing a data set by the collection of dyadic intervals of $\mathbb{R}$, i.e., intervals of the form $\left[k 2^{-n},(k+1) 2^{-n}\right)$, where $k$ and $n$ range over $Z$. These intervals are naturally nested by inclusion and can thus be organized according to a dyadic tree. In such a representation, a given dyadic interval has two child-nodes, namely the two half-intervals it is the union of. Gaussian stochastic processes (or random fields) indexed by the dyadic tree have been introduced in $[1,2]$ as an attempt to provide a statistical framework for mutiresolution signal processing. We refer the reader to $[1,2,3]$ for the motivation of this issue.

In particular, isotropic random fields have been investigated in detail in $[1,2]$. In these companion papers, two different kinds of problems are investigated:

1. How to parametrize such isotropic processes?

2. How to generate or synthesize them as outputs of some linear "filters" driven by white noise (the simplest isotropic process)?

A new form of "recursive" filters was introduced in $[1,2]$ for solving problem 2., a problem closely related with the construction of a Wold decomposition for the considered process. As usually, a Wold decomposition relies on some notion of "past" and "future". Such appropriate notions are introduced in [1] and are shown to correspond to coarser and finer scales respectively. Thus samples of isotropic processes are generated successively from coarse to fine scales. This orientation of the dyadic tree is also the basis for solving problem 1 in [1].

It is the main purpose of the present paper to show that the parametrization of isotropic processes can be obtained much more elegantly using a completely isometric point of view. Second, we extend the results of [1] to homogeneous trees of order $q$ ( $q=4$ corresponds to pyramidal data structures or quadtrees, used in image processing). And third, we provide a much more compact and elegant version of the Schur formulae which relate the process covariance sequence to its associated "reflection coefficient" sequence. Finally, we indicate how to recover easily the "oriented"

•IRISA/INRIA, Campus de Beaulieu, 35042 Rennes Cedex, France 
formulation of the results of [1] from the present unoriented ones.

The paper is organized as follows. In section 2 we introduce the basic concepts related to the isometric and the oriented point of view respectively. In section 3 we use the isometric point of view to define for isotropic processes a notion of prediction error vectors. These prediction errors are shown to satisfy a generalized Levinson recursion equation. Finally we give in section 3 a generalized Schur recursion, involving "one-dimensional" series in a formal variable $z$. This recursion relates the covariance sequence and the reflection coefficient sequence of the process. In section 4 we adopt the oriented point of view and define accordingly forward and backward prediction errors. The connection to the unoriented case is shown, and we give the Levinson recursions in the corresponding form (in the vector and in the barycentric version). We state a generalization of the Schur recursion of [1], involving formal series in "shifts on the tree," to the case of a tree of order $q$.

\section{The homogeneous tree of order $q$}

The homogeneous tree of order $q$ is an infinite acyclic nondirected connected graph such that every node has exactly $q+1$ branches to other nodes. By abuse of notation, we use $\mathcal{T}$ to denote both the tree and its collection of nodes. $\mathcal{T}$ has a natural notion of distance: $d(s, t)$ is the number of branches along the shortest path between the nodes $s, t \in \mathcal{T}$. This definition of $\mathcal{T}$ emphazises the isotropic nature of the tree: in the next subsection, we further develop this point of view. However, as we discussed in the introduction, the primary motivation for considering homogeneous trees is that they perfectly model pyramidal data structures: a second subsection is devoted to this alternative point of view.

\subsection{An isotropic point of view}

Let $s, t \in \mathcal{T}$ be two neighboring nodes, i.e. $d(s, t)=1$. Then we define a ball of diameter $n$ as follows:

for $n$ even:

$$
B_{n}(s, t) \triangleq\left\{u \in \mathcal{T} \mid d(u, s) \leq \frac{n}{2}\right\}
$$

for $n$ odd:

$$
\begin{aligned}
B_{n}(s, t) & \triangleq\left\{u \in \mathcal{T} \mid d(u, s) \leq \frac{n-1}{2} \vee d(u, t) \leq \frac{n-1}{2}\right\} \\
& =\left\{u \in \mathcal{T} \mid d(u, s) \leq \frac{n+1}{2} \wedge d(u, t) \leq \frac{n+1}{2}\right\}
\end{aligned}
$$

Note that the definition of $B_{n}(s, t)$ for even $n$ does not depend on $t$ so we shall often denote it simply by $B_{n}(s,$.$) . The following notion of a partial sphere will also be useful:$

$$
V_{n}(s, t) \triangleq\{u \in T \mid d(t, u)=n+1, d(s, u)=n\}
$$

Using the notion of a partial sphere, we can express relations between the balls $B_{n}(s, t)$ of different diameters: 
for $n$ even:

$$
\begin{aligned}
B_{n}(s, t) & =B_{n-1}(s, t) \cup V_{\frac{n}{2}}(s, t) \\
& =B_{n-1}(t, s) \cup V_{\frac{n}{2}}(s, t)
\end{aligned}
$$

for $n$ odd:

$$
\begin{aligned}
B_{n}(s, t) & =B_{n-1}(s, .) \cup V_{\frac{n-1}{2}}(t, s) \\
& =B_{n-1}(t, .) \cup V_{\frac{n-1}{2}}(s, t)
\end{aligned}
$$

Such objects are depicted in Figure 1.

\subsection{A pyramidal point of view}

We will introduce here an orientation on the tree. This alternative point of view enlightens the relations between the tree and its multiresolution interpretation.

\section{Geometry of the tree}

An important concept is the notion of a boundary point of a tree. Consider the set of infinite: sequences of $\mathcal{T}$ consisting of distinct nodes $t_{1}, t_{2}, \ldots$ where $d\left(t_{i}, t_{i+1}\right)=1$. A boundary point is an equivalence class of such sequences for the following equivalence relation: two sequences are equivalent if they differ only by a finite number of nodes. Let us select and fix one boundary point, denote it by $-\infty$. We can then define a notion of relative distance of two nodes with respect to $-\infty$ :

$$
\delta(s, t) \triangleq d(s, s \wedge t)-d(t, s \wedge t)
$$

where $s \wedge t$ is the node where the paths from $s$ and $t$ to $-\infty$ (i.e. the sequences $\left\{s_{i}\right\}_{i \in \mathbb{N}}$ with $s_{0}=s$ and $\left\{t_{i}\right\}_{i \in \mathbb{N}}$ with $t_{0}=t$ in the equivalence class $\left.-\infty\right)$ meet. This also yields an equivalence relation on nodes of $\mathcal{T}$ :

$$
s \asymp t \Leftrightarrow \delta(s, t)=0
$$

the equivalence classes for this relation are referred to as horocycles. This equivalence relation can be generalized to an order on the nodes of $\mathcal{T}$ : We write

$$
s \prec t \Leftrightarrow \delta(s, t)<0 .
$$

We can pick up the tree at $-\infty$ and let it hang from this boundary point: an horocycle will then appear as a horizontal plane as depicted in Figure 2. Finally, we shall call a horizontal clique a set of nodes located at the same horocyle, and lying at a distance 2 from each other (i.e. a clique is constituted by a set of $q$ nodes sharing the same parent-node). Horizontal cliques correspond in Figure 2 to triples located at the bottom of each elementary pyramide.

\subsubsection{Elementary moves on the tree}

To refer to nodes on the tree relatively to a particular node, it is useful to introduce the following moves on the tree (they act on the right on nodes, see Figure 2 for illustrations of these moves):

- 0 : no move 


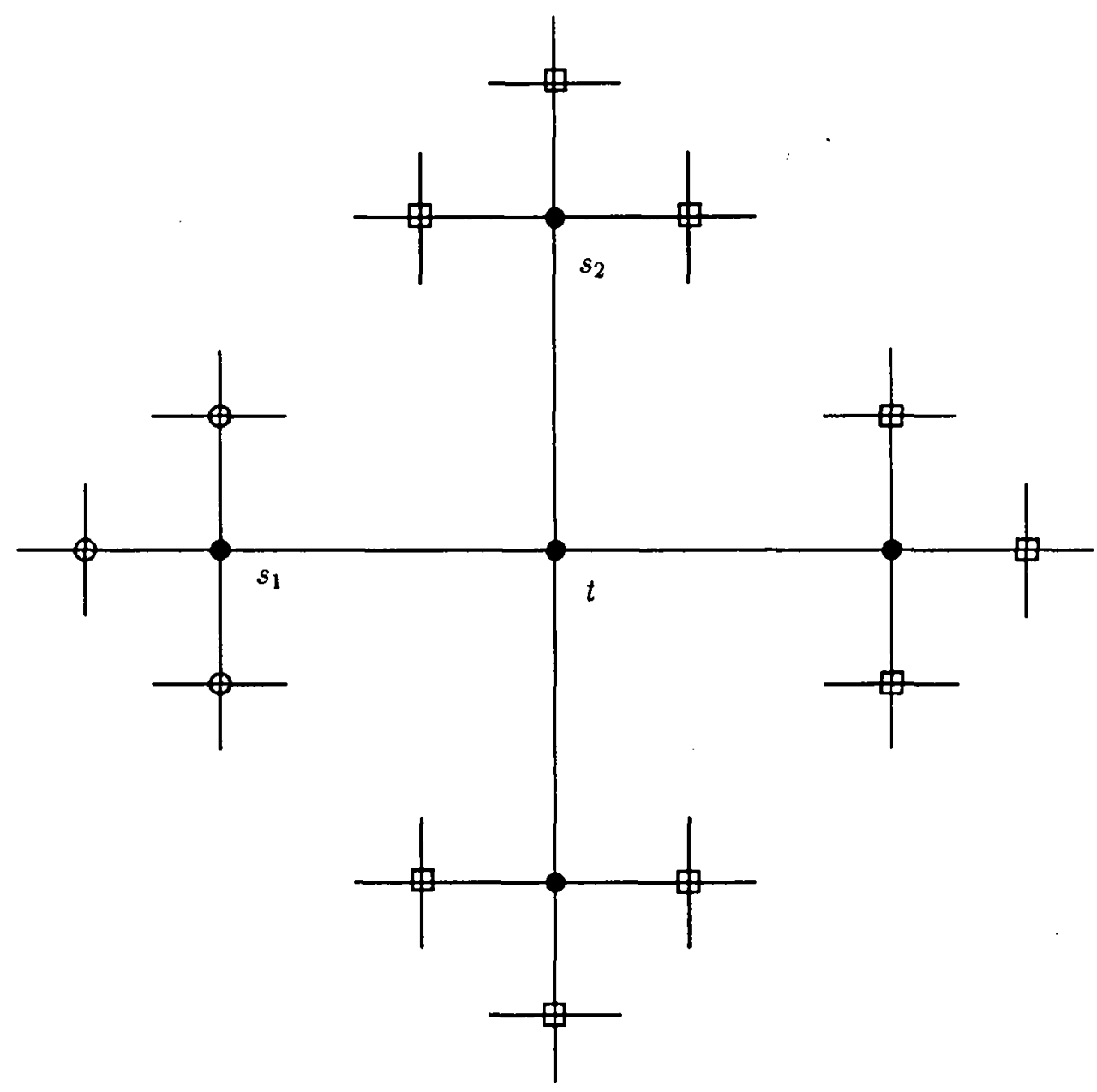

Figure 1: The tree of order $q=3$. The nodes "॰" represent the elements of the ball $B_{2}\left(t, s_{1}\right)=$ $B_{2}\left(t, s_{2}\right)$ of diameter 2 . The nodes "o" denote the set $V_{1}\left(s_{1}, t\right)$ and we have the relation $B_{2}\left(t, s_{1}\right) \cup$ $V_{1}\left(s_{1}, t\right)=B_{3}\left(t, s_{1}\right)=B_{3}\left(s_{1}, t\right)$. Furthermore, the nodes " $\square$ " mark the elements of the set $V_{2}\left(t, s_{1}\right)$, and we have, again, $B_{3}\left(t, s_{1}\right) \cup V_{2}\left(t, s_{1}\right)=B_{4}\left(t, s_{1}\right)=B_{4}\left(t, s_{2}\right)$. 
- $\gamma^{-1}$ : move one step toward $-\infty$

- $\delta_{(p)}$ : being finite sets, horizontal cliques can be totally ordered, select such an order for each horizontal clique and fix it from now on. Then $\delta_{(p)}$ maps $t$ to another point in the following manner: we move up the tree $p-1$ steps; we move to the successor of the so reached node in its horizontal clique (the successor of the last node in the clique is defined to be the first node of the same clique) and then descend $p-1$ steps; the steps for the descent are the same as the ones used for the ascent (i.e. if the last step in the ascent was from the $j$-th element of the clique to its parent-node $t$, then the first step in the descent will be from the successor of $t$ to the $j$-th node in the corresponding "child"-clique. We proceed that way for all remaining steps).

For each $p \in \mathbb{N} \backslash\{0\}$ we get thus $q-1$ different moves: $\delta_{(p)}, \delta_{(p)}^{2}, \ldots, \delta_{(p)}^{q-1}$, and we have clearly the relation $\delta_{(p)}^{q}=0$. 


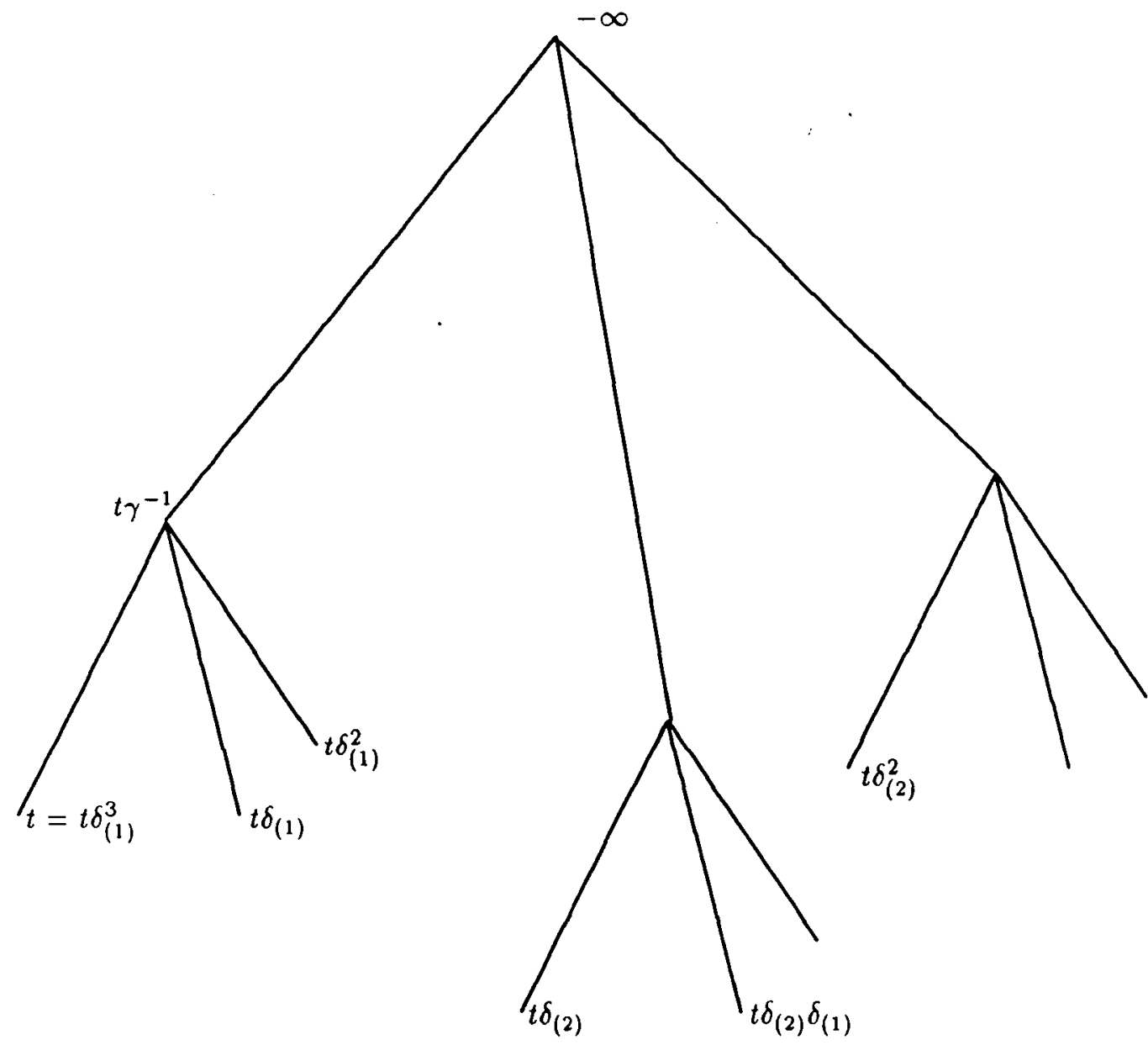

Figure 2: Illustration of moves on the hanging tree of order $q=3$. The set of nodes located at a same horizontal plane form a horocycle. The set $\left\{t, t \delta_{(1)}, t \delta_{(1)}^{2}\right\}$ is a set of $q$ nodes on one horocycle, lying at a distance of 2 to each other. These nodes share the same parent-node, they form a horizontal clique. 


\section{Parametrization of isotropic processes: an isotropic derivation}

We will focus here on isotropic processes. Recall that a zero-mean process $Y_{t}$ indexed by the nodes of the tree is said to be isotropic if its covariance function depends only on the distance between nodes:

$$
\mathbb{E}\left(Y_{t} Y_{s}\right)=r_{d(t, s)}
$$

Our objective here is to establish (in analogy with time series) Levinson and Schur recursions, leading to a representation of isotropic processes in terms of reflection coefficients.

\subsection{Levinson recursions}

Consider an isotropic process $Y_{t}$ on the homogeneous tree $\mathcal{T}$ of order $q$. For a given set $\mathcal{T}^{\prime} \subset \mathcal{T}$ we introduce

$$
\mathcal{Y}\left(\mathcal{T}^{\prime}\right)=\operatorname{span}\left\{Y_{t}, t \in \mathcal{T}^{\prime}\right\}
$$

and we write for short

$$
\mathcal{Y}_{n}(s, t) \triangleq \mathcal{Y}\left(B_{n}(s, t)\right)
$$

Using the notions of a ball $B_{n}$ and a partial sphere $V_{n}$ we can now define the prediction error $E_{n}$.

Definition 1 (prediction errors, prediction error spaces) For $u \in V_{\left\lfloor\frac{n}{2}\right\rfloor}(t, s)^{1}$ we define the $(n, t, s)$-prediction error (or prediction error for short when no confusion results) as being the following random variable:

$$
E_{n, t, s}(u) \triangleq Y_{u}-\mathbb{E}\left[Y_{u} \mid \mathcal{Y}_{n-1}(s, t)\right]
$$

and the corresponding prediction error space is

$$
\mathcal{E}_{n}(t, s) \triangleq \operatorname{span}\left\{E_{n, t, s}(u), u \in V_{\left\lfloor\frac{n}{2}\right\rfloor}(t, s)\right\} .
$$

We denote by $e_{n}(t, s)$ the barycenter of the components of $E_{n, t, s}$, i.e.

$$
e_{n}(t, s) \triangleq \frac{1}{\#} \sum_{u} E_{n, t, s}(u)
$$

where the symbol \# refers to the cardinal of the set $V_{\left\lfloor\frac{n}{2}\right\rfloor}(t, s)$. The partial sphere $V_{\left\lfloor\frac{n}{2}\right\rfloor}(t, s)$ will be referred to as the support of the considered prediction error.

These objects are depicted in Figure 3. The prediction errors allow us to state the following Levinson recursions, which are due to the fact that the union of a ball $B_{n}$ (of diameter $n$ ) and some associated partial sphere $V_{\left\lfloor\frac{n}{2}\right\rfloor}$ is again a ball, but of diameter $n+1$.

Theorem 1 (Levinson recursions) The prediction errors $E_{n, t, s}(u)$ satisfy the following recursions:

for $n$ even:

$$
E_{n+1, t, s}(u)=E_{n, t, s}(u)-\mathbb{E}\left[E_{n, t, s}(u) \mid \mathcal{E}_{n}(s, t)\right]
$$

for $n$ odd:

$$
E_{n+1, t, s}(u)=E_{n, t^{\prime}, t}(u)-\mathbb{E}\left[E_{n, t^{\prime}, t}(u) \mid \mathcal{E}_{n}(s, t)\right]
$$

where $t^{\prime}$ is such that

$$
d\left(t, t^{\prime}\right)+d\left(t^{\prime}, u\right)=d(t, u) \text { and } d\left(t, t^{\prime}\right)=1 .
$$

\footnotetext{
${ }^{1}$ for $x$ nonnegative, $\lfloor x\rfloor$ denotes the integer part of $x$, i.e., the greatest integer less than or equal to $x$.
} 

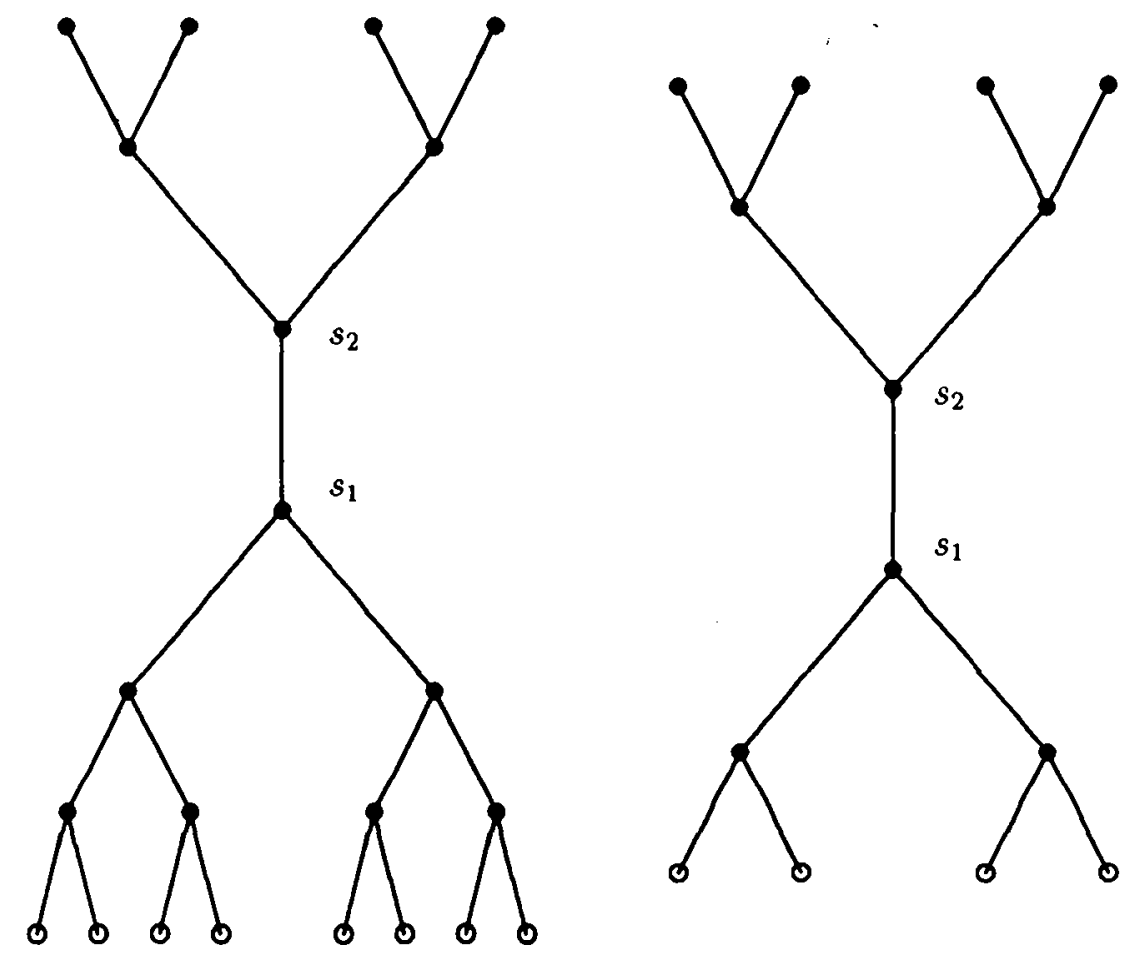

Figure 3: Prediction errors on the homogeneous tree $\mathcal{T}$ of order $q=2$. For $v \in V_{\left\lfloor\frac{n}{2}\right\rfloor}\left(s_{1}, s_{2}\right)$ the prediction error $E_{n, s_{1}, s_{2}}(v)$ is defined to be the residual of the projection of $Y_{v}$ on the space spanned by $Y_{t}, t \in B_{n-1}\left(s_{2}, s_{1}\right)$. In the case $n=6$ we have $B_{n-1}\left(s_{2}, s_{1}\right)=B_{5}\left(s_{2}, s_{1}\right)$ ("•") and $V_{\left\lfloor\frac{n}{2}\right\}}\left(s_{1}, s_{2}\right)=V_{3}\left(s_{1}, s_{2}\right)$ ("०"). For $n=5$ we have the corresponding $B_{4}\left(s_{2}, s_{1}\right)$ ("•") and $V_{2}\left(s_{1}, s_{2}\right)$ ("O"). 
Proof: Using the relations (4),(5) and using the Definition (8) of $E_{n, t, s}(u)$, we get easily for $n$ even:

$$
\mathcal{Y}_{n}(s, t)=\mathcal{Y}_{n-1}(s, t) \oplus \mathcal{E}_{n}(s, t)
$$

for $n$ odd:

$$
\mathcal{Y}_{n}(s, t)=\mathcal{Y}_{n-1}\left(t, t^{\prime}\right) \oplus \mathcal{E}_{n}(s, t) .
$$

Then, inserting these expressions in the definition of $E_{n, t, s}(u)$, the result follows immediately.

By inspecting carefully the supports of the prediction errors of successive orders, we obtain the following vector-equations:

for $n$ even:

$$
E_{n+1, t, s}=E_{n, t, s}-\mathbb{E}\left[E_{n, t, s} \mid \mathcal{E}_{n}(s, t)\right]
$$

for $n$ odd:

$$
E_{n+1, t, s}=\left(\begin{array}{c}
E_{n, t_{1}, t} \\
E_{n, t_{2}, t} \\
\vdots \\
E_{n, t_{q}, t}
\end{array}\right)-\mathbf{E}\left[\left(\begin{array}{c}
E_{n, t_{1}, t} \\
E_{n, t_{2}, t} \\
\vdots \\
E_{n, t_{q}, t}
\end{array}\right) \mid \mathcal{E}_{n}(s, t)\right],
$$

where $\left\{s, t_{1}, t_{2}, \ldots, t_{q}\right\}$ is the set of all neighboring nodes of $t$. (We supposed here that the elements of the vectors are in an appropriate order, i.e. if we have $E_{n, t_{i}, t}=\left(E_{n, t_{t}, t}\left(u_{i 1}\right), \ldots, E_{n, t_{i}, t}\left(u_{i n}\right)\right)^{T}$ then $E_{n+1, t, s}$ is of the form $E_{n+1, t, s}=\left(E_{n+1, t, s}\left(u_{11}\right), E_{n+1, t, s}\left(u_{12}\right), \ldots, E_{n+1, t, s}\left(u_{1 n}\right), E_{n+1, t, s}\left(u_{21}\right), \ldots\right)^{T}$. Using lemma 7 of Appendix A, we obtain immediately

for $n$ even:

$$
E_{n+1, t, s}=E_{n, t, s}-\mathbb{E}\left[E_{n, t, s} \mid e_{n}(s, t)\right]
$$

for $n$ odd:

$$
E_{n+1, t, s}=\left(\begin{array}{c}
E_{n, t_{1}, t} \\
E_{n, t_{2}, t} \\
\vdots \\
E_{n, t_{q}, t}
\end{array}\right)-\mathbb{E}\left[\left(\begin{array}{c}
E_{n, t_{1}, t} \\
E_{n, t_{2}, t} \\
\vdots \\
E_{n, t_{q}, t}
\end{array}\right) \mid e_{n}(s, t)\right] .
$$

These recursions involve the conditional expectations based on the scalars $e_{n}(s, t)$ instead of the spaces $\mathcal{E}_{n}(s, t)$. It turns out that these latter recursions do require more work for their proof. In Appendix $\mathrm{A}$ we develop some results in a very general context, which allow us to establish these recursions.

\subsection{Levinson recursions for the barycenters}

By taking the barycenters of the vectors involved in recursions (13),(14), we obtain the following recursions involving only the barycenters $e_{n}$ :

Lem ma 1 The barycenters of the prediction errors satisfy the recursions for $n$ even:

$$
\left.e_{n+1}(t, s)=e_{n}(t, s)-\mathbb{E}\left[e_{n}(t, s) \mid e_{n}(s, t)\right)\right]
$$

for $n$ odd:

$$
e_{n+1}(t, s)=\frac{1}{q} \sum_{i=1}^{q} e_{n}\left(t_{i}, t\right)-\frac{1}{q} \mathbb{E}\left[\sum_{i=1}^{q} e_{n}\left(t_{i}, t\right) \mid e_{n}(s, t)\right]
$$


Using appropriate isometries, we show in Appendix A that $\mathbb{E}\left[e_{n}(t, s) e_{n}(s, t)\right]$ and $\mathbb{E}\left[e_{n}\left(t_{i}, t\right) e_{n}(s, t)\right]$ are constants which do not depend on a particular choice of the neighboring nodes $s, t, t_{i}$, and we can thus state the following theorem, where we introduce the reflection coefficients $k_{n}$.

\section{Theorem 2 (barycentric Levinson recursions).}

for $n$ even:

$$
e_{n+1}(t, s)=e_{n}(t, s)-k_{n+1} e_{n}(s, t)
$$

where the reflection coefficient (or PARCOR) $k_{n+1}$ is given by

$$
k_{n+1}=\operatorname{cor}\left(e_{n}(t, s), e_{n}(s, t)\right)
$$

for $n$ odd:

$$
e_{n+1}(t, s)=\frac{1}{q} \sum_{i=1}^{q} e_{n}\left(t_{i}, t\right)-k_{n+1} e_{n}(s, t)
$$

where the reflection coefficient $k_{n+1}$ is given by

$$
k_{n+1}=\operatorname{cor}\left(e_{n}\left(t_{1}, t\right), e_{n}(s, t)\right) .
$$

Corollary 1 The variances $\sigma^{2}(n)$ of $e_{n}(t, s)$ satisfy the following recursions: for $n$ even:

$$
\sigma^{2}(n+1)=\left(1-k_{n+1}^{2}\right) \sigma^{2}(n)
$$

so that the following inequalities hold:

$$
-1 \leq k_{n+1} \leq 1
$$

for $n$ odd:

$$
\sigma^{2}(n+1)=\left(\frac{1}{q}+\frac{q-1}{q} k_{n+1}-k_{n+1}^{2}\right) \sigma^{2}(n)
$$

so that the following inequalities hold:

$$
-\frac{1}{q} \leq k_{n+1} \leq 1
$$

Proof: The equations (17),(19) are immediate consequences of (15),(16) and the coefficients $k_{n+1}$ can be calculated in the following way:

for $n$ even:

$$
\begin{aligned}
k_{n+1} & =\frac{1}{\sigma^{2}(n)} \mathbf{E}\left(e_{n}(t, s) e_{n}(s, t)\right) \\
& =\operatorname{cor}\left(e_{n}(t, s), e_{n}(s, t)\right)
\end{aligned}
$$

hence we have

$$
\mathbb{E}\left(e_{n}(t, s) e_{n}(s, t)\right)=k_{n+1} \sigma^{2}(n)
$$

and using (17) we get

$$
\begin{aligned}
\sigma^{2}(n+1) & =\mathbb{E}\left(e_{n+1}(t, s) e_{n+1}(t, s)\right) \\
& =\sigma^{2}(n)+k_{n+1}^{2} \sigma^{2}(n)-2 k_{n+1} \mathbb{E}\left(e_{n}(t, s) e_{n}(s, t)\right) \\
& \stackrel{(25)}{=}\left(1-k_{n+1}^{2}\right) \sigma^{2}(n)
\end{aligned}
$$


Finally, taking into account the constraint $\sigma^{2}(n+1) \geq 0$, we get (22).

for $n$ odd:

$$
\begin{aligned}
k_{n+1} & =\frac{1}{q \sigma^{2}(n)} \mathbb{E}\left(\left(\sum_{i=1}^{q} e_{n}\left(t_{i}, t\right)\right) e_{n}(s, t)\right) \\
& =\frac{1}{q \sigma^{2}(n)} q \mathbb{E}\left(e_{n}\left(t_{1}, t\right) e_{n}(s, t)\right) \\
& =\operatorname{cor}\left(e_{n}\left(t_{1}, t\right), e_{n}(s, t)\right)
\end{aligned}
$$

and, using (19) we obtain

$$
\begin{aligned}
\sigma^{2}(n+1)= & \left(\frac{1}{q}+k_{n+1}^{2}\right) \sigma^{2}(n)+q(q-1) \frac{1}{q^{2}} \mathbb{E}\left(e_{n}(s, t) e_{n}\left(t_{1}, t\right)\right) \\
& -2 q k_{n+1} \frac{1}{q}\left(\mathbb{E}\left(e_{n}(s, t) e_{n}\left(t_{1}, t\right)\right)\right. \\
\stackrel{(26)}{=} & \left(\frac{1}{q}+k_{n+1}^{2}\right) \sigma^{2}(n)+\left(\frac{q-1}{q}-2 k_{n+1}\right) k_{n+1} \sigma^{2}(n) \\
= & \left(\frac{1}{q}+\frac{q-1}{q} k_{n+1}-k_{n+1}^{2}\right) \sigma^{2}(n) .
\end{aligned}
$$

Recalling again the constraint $\sigma^{2}(n+1) \geq 0$, we finally get (24).

\subsection{Schur-recursions}

In analogy to the case of time-indexed processes, where the Schur-recursions involve formal series in $z$ ( $z$ being the backwards-shift operator), one would expect here recursions involving formal series in "shifts on the tree" (encoded by using the $\delta$ and $\gamma^{-1}$ shifts, cf. Section 2.2.1). In Section 4.3 we will explicitly give Schur-recursions in that mentioned form. Due to the isotropy of the considered processes it is however possible to establish Schur-recursions involving "one-dimensional" formal series, which we will encode in terms of a formal variable $z$. For this purpose we chose an arbitrary path $\left\{t_{i}\right\}_{i \in Z}$ on the tree $\mathcal{T}$, i.e. we have $t_{i} \neq t_{j}$ if $i \neq j$ and $d\left(t_{i}, t_{i+1}\right)=1 \forall i$. We introduce $\forall i$ the notation $\left\{t_{i+\delta}, j=1, \ldots, q-1\right\}$ to be the set of neighboring nodes of $t_{i}$ which are not elements of the chosen path, i.e. we have $d\left(t_{i}, t_{i+\delta}\right)=1, t_{i-1} \neq t_{i+\delta}, \neq t_{i+1}$. We define thus the following series in $z$,

$$
\begin{aligned}
& P_{n} \triangleq \sum_{i \geq 0} \mathbb{E}\left(Y_{t_{0}} e_{n}\left(t_{\left\lfloor\frac{n}{2}\right\rfloor+i}, t_{\left\lfloor\frac{n}{2}\right\rfloor+i+1}\right)\right) z^{-i} \forall n \geq 0 \\
& Q_{n} \triangleq \sum_{i \geq 0} \mathbb{E}\left(Y_{t_{0}} e_{n}\left(t_{\left\lfloor\frac{n+1}{2}\right\rfloor+i}, t_{\left\lfloor\frac{n+1}{2}\right\rfloor+i-1}\right)\right) z^{-i} \forall n \geq 1 \\
& Q_{0} \triangleq \sum_{i \geq 1} \mathbb{E}\left(Y_{t_{0}} e_{0}\left(t_{i}, t_{i-1}\right)\right) z^{-i},
\end{aligned}
$$

which allow us to introduce a generalized Schur-recursion. We can use Lemma 8 (cf. Appendix A) to show that $P_{n}$ and $Q_{n}$ are well-defined, i.e. do not depend on the choice of the path $\left\{t_{i}\right\}_{i \in Z}$. We denote by $P_{n}(i), Q_{n}(i)$ the coefficient associated to $z^{-i}$ in $P_{n}, Q_{n}$ respectively. 
Theorem 3 (Schur-recursions) Let $P_{0}=\sum_{i \geq 0} z^{-i} r(i), Q_{0}=\sum_{i \geq 1} z^{-i} r(i)$. Then we have the following recursion-equations:

$n$ odd:

$$
\begin{aligned}
P_{n} & =P_{n-1}-k_{n} z Q_{n-1} \\
Q_{n} & =z Q_{n-1}-k_{n} P_{n-1}
\end{aligned}
$$

where

$$
k_{n}=\frac{Q_{n-1}(1)}{P_{n-1}(0)}
$$

$n$ even:

$$
\begin{aligned}
P_{n} & =\frac{1}{q} P_{n-1}+\left(\frac{q-1}{q}-k_{n}\right) z Q_{n-1} \\
Q_{n} & =z Q_{n-1}-k_{n} P_{n-1}
\end{aligned}
$$

where

$$
k_{n}=\frac{Q_{n-1}(1)}{P_{n-1}(0)}
$$

Using these equations we are able to compute recursively the series $P_{n}, Q_{n}$ as well as the reflection coefficients $k_{n}$.

Proof: The initial conditions are easily verified by noting the fact that $e_{0}(t, s)=E_{0, t, s}(t)=Y_{t}$. In order to prove the recursion equations we will first consider the elementary expressions of the form $\mathbb{E}\left(Y_{t_{0}} e_{n}(.,).\right)$, which appear in the definitions (27),(28). We will make use of the fact that $\mathbb{E}\left(Y_{t} e_{n}\left(s_{1}, s_{2}\right)\right)$ depends only on the distance $d\left(t, s_{1}\right)$ and on the relative orientation of the branch $\left(s_{1}, s_{2}\right)$ with respect to $t$, i.e. $\mathbb{E}\left(Y_{t} e_{n}\left(s_{1}, s_{2}\right)\right)$ depends on $d\left(t, s_{1}\right)$ and the sign of $\left(d\left(t, s_{1}\right)-d\left(t, s_{2}\right)\right)$. This is shown in Appendix A.2 using an argument of isometries.

In the case $n$ odd we use equation (17) to obtain the following relations

$$
\begin{aligned}
\mathbb{E}\left(Y_{t_{0}} e_{n}\left(t_{i}, t_{i+1}\right)\right) & =\mathbb{E}\left(Y_{t_{0}}\left(e_{n-1}\left(t_{i}, t_{i+1}\right)-k_{n} e_{n-1}\left(t_{i+1}, t_{i}\right)\right)\right) \\
& =\mathbb{E}\left(Y_{t_{0}} e_{n-1}\left(t_{i}, t_{i+1}\right)\right)-k_{n} \mathbb{E}\left(Y_{t_{0}} e_{n-1}\left(t_{i+1}, t_{i}\right)\right) \\
& \\
\mathbb{E}\left(Y_{t_{0}} e_{n}\left(t_{i+1}, t_{i}\right)\right) & =\mathbb{E}\left(Y_{t_{0}}\left(e_{n-1}\left(t_{i+1}, t_{i}\right)-k_{n} e_{n-1}\left(t_{i}, t_{i+1}\right)\right)\right) \\
& =\mathbb{E}\left(Y_{t_{0}} e_{n-1}\left(t_{i+1}, t_{i}\right)\right)-k_{n} \mathbb{E}\left(Y_{t_{0}} e_{n-1}\left(t_{i}, t_{i+1}\right)\right)
\end{aligned}
$$

In the case $n$ even we use equation (19) and obtain for $i \geq 1$ the equation

$$
\begin{aligned}
& \mathbb{E}\left(Y_{t_{0}} e_{n}\left(t_{i}, t_{i+1}\right)\right) \\
= & \mathbb{E}\left(Y_{t_{0}}\left(\frac{1}{q} e_{n-1}\left(t_{i-1}, t_{i}\right)+\frac{1}{q} \sum_{j=1}^{q-1} e_{n-1}\left(t_{i+\delta_{j}}, t_{i}\right)-k_{n} e_{n-1}\left(t_{i+1}, t_{i}\right)\right)\right) \\
= & \frac{1}{q} \mathbb{E}\left(Y_{t_{0}} e_{n-1}\left(t_{i-1}, t_{i}\right)\right)+\left(\frac{q-1}{q}-k_{n}\right) \mathbb{E}\left(Y_{t_{0}} e_{n-1}\left(t_{i+1}, t_{i}\right)\right)
\end{aligned}
$$


and for $i \geq 0$ we have:

$$
\begin{aligned}
& \mathbb{E}\left(Y_{t_{0}} e_{n}\left(t_{i+1}, t_{i}\right)\right) \\
= & \mathbb{E}\left(Y_{t_{0}}\left(\frac{1}{q} e_{n-1}\left(t_{i+2}, t_{i+1}\right)+\frac{1}{q} \sum_{j=1}^{q-1} e_{n-1}\left(t_{i+1+\delta_{j}}, t_{i+1}\right)-k_{n} e_{n-1}\left(t_{i}, t_{i+1}\right)\right)\right) \\
= & \mathbb{E}\left(Y_{t_{0}} e_{n-1}\left(t_{i+2}, t_{i+1}\right)\right)-k_{n} \mathbb{E}\left(Y_{t_{0}} e_{n-1}\left(t_{i}, t_{i+1}\right)\right)
\end{aligned}
$$

Inserting these equations (36)-(39) into the definitions (27),(28) of $P_{n}$ and $Q_{n}$ we obtain the following relations

$n$ odd:

$$
\begin{aligned}
P_{n} & =\sum_{i \geq 0}\left(\mathbb{E}\left(Y_{t_{0}} e_{n-1}\left(t_{\frac{n-1}{2}+i}, t_{\frac{n+1}{2}+i}\right)\right)-k_{n} \mathbb{E}\left(Y_{t_{0}} e_{n-1}\left(t_{\frac{n+1}{2}+i}, t_{\frac{n-1}{2}+i}\right)\right)\right) z^{-i} \\
& =P_{n-1}-k_{n} z Q_{n-1}+k_{n} z Q_{n-1}(0) \\
& =P_{n-1}-k_{n} z Q_{n-1}
\end{aligned}
$$

where we used the fact that, by definition, $Q_{i}(0)=0$ (this follows easily by noting the fact that $e_{n}\left(t_{\left\lfloor\frac{n+1}{2}\right\rfloor}, t_{\left\lfloor\frac{n+1}{2}\right\rfloor-1}\right)$ represents the barycenter of the prediction error based on the observations of $Y_{t}^{\prime}, t \in B_{n-1}\left(t_{\left\lfloor\frac{n+1}{2}\right\rfloor-1}, t_{\left\lfloor\frac{n+1}{2}\right\rfloor}\right)$, and since $Y_{t_{0}} \in B_{n-1}\left(t_{\left\lfloor\frac{n+1}{2}\right\rfloor-1}, t_{\left\lfloor\frac{n+1}{2}\right\rfloor}\right)$ we have $\left.\mathbb{E}\left(Y_{t_{0}} e_{n}\left(t_{\left\lfloor\frac{n+1}{2}\right\rfloor}, t_{\left\lfloor\frac{n+1}{2}\right\rfloor-1}\right)\right)=0\right)$. Starting with the definition of $Q_{n}$ we obtain

$$
\begin{aligned}
Q_{n} & =\sum_{i \geq 0}\left(\mathbb{E}\left(Y_{t_{0}} e_{n-1}\left(t_{\frac{n+1}{2}+i}, t_{\frac{n-1}{2}+i}\right)-k_{n} \mathbb{E}\left(Y_{t_{0}} e_{n-1}\left(t_{\frac{n-1}{2}+i}, t_{\frac{n+1}{2}+i}\right)\right)\right) z^{-i}\right. \\
& =z Q_{n-1}-k_{n} P_{n-1}
\end{aligned}
$$

Proceeding in the same way for $n \operatorname{even}(n \geq 2)$ we obtain

$$
\begin{aligned}
P_{n} & =\sum_{i \geq 0}\left(\frac{1}{q} \mathbb{E}\left(Y_{t_{0}} e_{n-1}\left(t_{\frac{n}{2}+i-1}, t_{\frac{n}{2}+i}\right)\right)+\left(\frac{q-1}{q}-k_{n}\right) \mathbb{E}\left(Y_{t_{0}} e_{n-1}\left(t_{\frac{n}{2}+i+1}, t_{\frac{n}{2}+i}\right)\right)\right) z^{-i} \\
& =\frac{1}{q} P_{n-1}+\left(\frac{q-1}{q}-k_{n}\right) z Q_{n-1}-\left(\frac{q-1}{q}-k_{n}\right) z Q_{n-1}(0) \\
& =\frac{1}{q} P_{n-1}+\left(\frac{q-1}{q}-k_{n}\right) z Q_{n-1}
\end{aligned}
$$

and

$$
\begin{aligned}
Q_{n} & =\sum_{i \geq 0}\left(\mathbb{E}\left(Y_{t_{0}} e_{n-1}\left(t_{\frac{n}{2}+1+i}, t_{\frac{n}{2}+i}\right)\right)-k_{n} \mathbb{E}\left(Y_{t_{0}} e_{n-1}\left(t_{\frac{n}{2}+i-1}, t_{\frac{n}{2}+i}\right)\right)\right) z^{-i} \\
& =z Q_{n-1}-k_{n} P_{n-1} .
\end{aligned}
$$

This finishes the proof of the recursion equations (30), (31) and (33),(34). The equations which enable us to compute the reflection coefficients $k_{n}$ are obtained by using just the equation $Q_{n}=$ $z Q_{n-1}-k_{n} P_{n-1}$ and the fact that $Q_{i}(0)=0 \forall i$. 


\section{Levinson and Schur recursions - the pyramidal point of view}

\subsection{Forward and Backward Prediction Errors and Levinson Recursions}

We adopt in the following the pyramidal point of view. Each causal move on the tree (causal in the sense that it is a move from a node $t$ to the same horocycle as $t$ or to a previous horocycle) can be encoded by words using the elementary moves $\gamma^{-1}, \delta_{(p)}$ as alphabet. It is easily verified, that the $\delta$-operators commute with each other, but they do not commute with the $\gamma^{-1}$-operator. Note that, due to that non-commutativity, for given nodes $s, t$ with $s \prec t$ the word $\omega$ such that $s=t \omega$ is not uniquely determined. Thus we generate a set $\mathcal{L}$ of words $\omega$ on $\mathcal{T}$. The language $\mathcal{L}$ is supplied with a natural notion of length. For all $\omega \in \mathcal{L}$ we define

$$
|\omega| \triangleq d(t, t \omega) \text {. }
$$

In order to distinguish between the words $\omega$ we introduce the notations $\omega \prec 0, \omega \asymp 0$ if $\delta(t \omega, t)<0$, $\delta(t \omega, t)=0$ respectively.

\subsubsection{Prediction Errors}

In analogy with the time series case, the development of prediction error models is based upon the construction of the past of a node $t$. We define here the following spaces, representing the past of order $n$ of the process $Y$ with respect to a given node $t \in \mathcal{T}$ :

$$
\mathcal{Y}_{t, n} \triangleq \operatorname{span}\left\{Y_{t \omega}, \omega \in \mathcal{L},|\omega| \leq n\right\}
$$

In a multiresolution framework, an horocycle constitutes a certain scale, the previous horocycles correspond to coarser scales. Therefore, the past $\mathcal{Y}_{t, n}$ of a node $t$ is a space spanned by the values of the process $Y$ evaluated at the same or at coarser scales. We will now define the forward and backward prediction errors:

Definition 2 (backward prediction error, backward prediction error space) For $\omega \in \mathcal{L}$ such that $|\omega|=n$, we define the backward prediction error to be the following random variable:

$$
F_{t, n}(\omega) \triangleq Y_{t \omega}-\mathbb{E}\left[Y_{t \omega} \mid \mathcal{Y}_{t, n-1}\right]
$$

The corresponding backward prediction error space is defined by

$$
\mathcal{F}_{t, n} \triangleq \operatorname{span}\left\{F_{t, n}(\omega)|\omega \in \mathcal{L},| \omega \mid=n\right\}
$$

A simple calculation shows that

$$
\operatorname{dim} F_{t, n}=q^{\left\lfloor\frac{n}{2}\right\rfloor} .
$$

One of the basic ideas of the Levinson recursions is the interpretation of $\mathcal{F}_{t, n}$ as an orthogonal complement:

$$
\mathcal{Y}_{t, n}=\mathcal{Y}_{t, n-1} \oplus \mathcal{F}_{t, n}
$$


Definition 3 (forward prediction error, forward prediction error space) For $n \geq 1$, for $\omega \in \mathcal{L}$ such that $\omega \asymp 0,|\omega| \leq n-1$, we define the forward prediction error to be the following random variable:

$$
E_{t, n}(\omega) \triangleq Y_{t \omega}-\mathbb{E}\left[Y_{t \omega} \mid \mathcal{Y}_{t \gamma^{-1}, n-1}\right],
$$

for $n=0$ we define $E_{t, 0}(0) \triangleq Y_{t}$. The corresponding forward prediction error space is

$$
\mathcal{E}_{t, n} \triangleq \operatorname{span}\left\{E_{t, n}(\omega)|\omega \in \mathcal{L},| \omega \mid \leq n-1 \text { and } \omega \asymp 0\right\} .
$$

The dimension of the forward prediction error is easily verified to be

$$
\operatorname{dim} E_{t, n}=q^{\left\lfloor\frac{n-1}{2}\right\rfloor} .
$$

The representation of $\mathcal{E}_{t, n}$ as an orthogonal complement requires the distinction between two cases: $n$ odd :

$$
\mathcal{Y}_{t, n} \ominus \mathcal{Y}_{t \gamma^{-1}, n-1}=\mathcal{E}_{t, n}
$$

$n$ even :

$$
\mathcal{Y}_{t, n} \ominus \mathcal{Y}_{t \gamma-1, n-1}=\mathcal{E}_{t, n}+\mathcal{E}_{t \delta_{\left(\frac{n}{2}\right), n}}+\ldots+\mathcal{E}_{t \delta_{\left(\frac{n}{2}\right)}^{q-1}, n}
$$

(note that on the righthand side of equation (49) we do not have an orthogonal sum). A crucial point is to note that the spaces and prediction errors defined here already appear in Section 3; more precisely, the following equalities hold:

$$
\begin{aligned}
\mathcal{Y}_{t, n} & =\mathcal{Y}_{n}\left(t \gamma^{-\left\lfloor\frac{n}{2}\right\rfloor}, t \gamma^{-\left\lfloor\frac{n}{2}\right\rfloor-1}\right) \\
& =\mathcal{Y}_{n}\left(t \gamma^{-\left\lfloor\frac{n+1}{2}\right\rfloor}, t \gamma^{-\left\lfloor\frac{n+1}{2}\right\rfloor+1}\right)
\end{aligned}
$$

Using (50),(51) for the backward and forward prediction error respectively, we find the equalities (cf. (8))

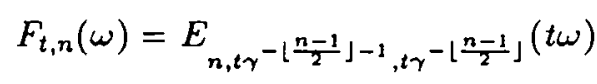

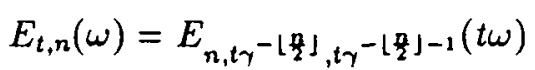

which establish the connection between the forward and backward prediction errors and the "isotropic" prediction error used throughout section 3 .

\subsubsection{Levinson Recursions}

We will develop now a set of Levinson recursion equations; they are established using the corresponding recursions $(9),(10)$ of the previous section, but we have to distinguish here between the backward and forward prediction errors $F_{t, n}(\omega)$ and $E_{t, n}(\omega)$.

$n$ even: Let us consider first $F_{t, n}(\omega)$ which is defined for $|\omega|=n$; there are two subclasses of such words $\omega$. 
Case $1: \omega \prec 0$; then $\omega$ is of the form $\omega=\gamma^{-1} \tilde{\omega}$, with $|\tilde{\omega}|=n-1$. Equation (52) applied for an even integer $n$ yields

$$
F_{t, n}(\omega)=E_{n, t \gamma}-\frac{n}{2}, t \gamma^{-\frac{n}{2}+1}(t \omega) .
$$

Writing the unoriented Levinson recursion (10) (for the order $n^{\prime}=n-1$, odd), we get:

$$
E_{n, t \gamma}-\frac{n}{2}, t \gamma^{-\frac{n}{2}+1}(t \omega)=E_{n-1, t^{\prime}, t \gamma^{-\frac{n}{2}}}(t \omega)-\mathbb{E}\left[E_{n-1, t^{\prime}, t \gamma^{-\frac{n}{2}}}(t \omega) \mid \mathcal{E}_{n-1}\left(t \gamma^{-\frac{n}{2}+1}, t \gamma^{-\frac{n}{2}}\right)\right]
$$

The critical point is the location of the node $t^{\prime}$, which is shown to be

$$
t^{\prime}=t \gamma^{-\frac{n}{2}-1} \text {. }
$$

Using (52) and (53), we get easily

$$
\begin{aligned}
E_{n-1, t \gamma^{-\frac{n}{2}-1}, t \gamma^{-\frac{n}{2}}}(t \omega) & =F_{t \gamma^{-1}, n-1}(\tilde{\omega}) \\
\mathcal{E}_{n-1}\left(t \gamma^{-\frac{n}{2}+1}, t \gamma^{-\frac{n}{2}}\right) & =\mathcal{E}_{t, n-1}
\end{aligned}
$$

from which we deduce that

$$
F_{t, n}(\omega)=F_{t \gamma^{-1}, n-1}(\tilde{\omega})-\mathbb{E}\left[F_{t \gamma^{-1}, n-1}(\tilde{\omega}) \mid \mathcal{E}_{t, n-1}\right]
$$

Case $2: \omega \asymp 0$. Since $|\omega|=n$, we see that there exists $p$ with $1 \leq p \leq q-1$, and $\tilde{\omega}$ with $|\bar{\omega}|<n, \bar{\omega} \asymp 0$ such that $\omega$ can be rewritten in the form

$$
\omega=\delta_{\left(\frac{n}{2}\right)}^{p} \tilde{\omega}
$$

By using the same corresponding unoriented recursion (10) as before, we obtain again equation (54), but in this case we have

$$
t^{\prime}=t \gamma^{-\frac{n}{2}+1} \delta_{(1)}^{p}
$$

Using (52) we get

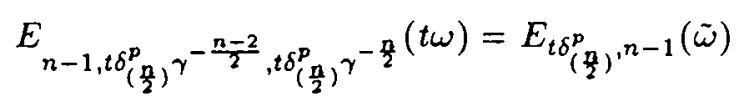

Now the geometry of the tree provides the equalities

$$
\begin{aligned}
t \delta_{\left(\frac{n}{2}\right)}^{p} \gamma^{-\frac{n}{2}+1} & =t \gamma^{-\frac{n}{2}+1} \delta_{(1)}^{p} \\
t \delta_{\left(\frac{n}{2}\right)}^{p} \gamma^{-\frac{n}{2}} & =t \gamma^{-\frac{n}{2}} .
\end{aligned}
$$

(these are due to the non-commutativity of the words $\delta_{(p)}$ and $\gamma^{-1}$ ). Therefore, (54) yields in this case:

$$
F_{t, n}(\omega)=E_{t \delta_{\left(\frac{n}{2}\right)}^{p}, n-1}(\tilde{\omega})-\mathbb{E}\left[E_{t \delta_{\left(\frac{n}{2}\right)}^{p}, n-1}(\tilde{\omega}) \mid \mathcal{E}_{t, n-1}\right]
$$

The proofs of the remaining cases (recursion for $E_{t, n}, n$ even, and the recursions for $n$ odd) are obtained in a similar way, they are left to the reader. We will just state the results. We consider now $E_{t, n}(\omega)$ which is defined for $\omega \asymp 0$ and $|\omega|<n$ and we obtain

$$
E_{t, n}(\omega)=E_{t, n-1}(\omega)-\mathbb{E}\left(E_{t, n-1}(\omega) \mid \mathcal{F}_{t \gamma^{-1}, n-1}\right) .
$$


$n$ odd : For $n=1$, we get the starting point of our recursions,

$$
\begin{aligned}
& F_{t, 1}=Y_{t \gamma^{-1}}-k_{1} Y_{t} \\
& E_{t, 1}=Y_{t}-k_{1} Y_{t \gamma^{-1}}
\end{aligned}
$$

where $k_{1}$ is the first reflection coefficient, given by

$$
k_{1}=\frac{\mathbb{E}\left(Y_{t \gamma^{-1}} Y_{t}\right)}{\mathbf{E}\left(Y_{t \gamma^{-1}}^{2}\right)}
$$

Consider next the case of $F_{t, n}(\omega)$ for $n \geq 3$ where we have (by definition) $|\omega|=n$. Such words $\omega$ are of the following form (recall that $n$ is odd)

$$
\omega=\gamma^{-1} \tilde{\omega},|\tilde{\omega}|=n-1 .
$$

We get thus

$$
F_{t, n}(\omega)=F_{t \gamma^{-1}, n-1}(\tilde{\omega})-\mathbb{E}\left[F_{t \gamma^{-1}, n-1}(\tilde{\omega}) \mid \mathcal{E}_{t, n-1}, \mathcal{E}_{t \delta_{\left(\frac{n-1}{2}\right.}, n-1}, \ldots, \mathcal{E}_{t \delta_{\left(\frac{n-1}{2}\right)}^{q-1}, n-1}\right] .
$$

Consider now the computation of $E_{t, n}(\omega)$ for $n \geq 3$ where we have $|\omega|<n$ and $\omega \asymp 0$. Again, we have to distinguish between two classes of such $\omega$ :

Case 1: $|\omega|<n-1$, we obtain

$$
E_{t, n}(\omega)=E_{t, n-1}(\omega)-\mathbb{E}\left[E_{t, n-1}(\omega) \mid \mathcal{F}_{t \gamma^{-1}, n-1}\right]
$$

Case 2: $|\omega|=n-1$, there exists $p$ with $1 \leq p \leq q-1$ and $\tilde{\omega}$ with $\tilde{\omega} \asymp 0$ such that

$$
\omega=\delta_{\left(\frac{n-1}{2}\right)}^{p} \tilde{\omega} \text {. }
$$

We get thus

$$
E_{t, n}(\omega)=E_{t \delta_{\left(\frac{n-1}{2}\right)}^{p}, n-1}(\tilde{\omega})-\mathbb{E}\left[E_{t \delta_{\left(\frac{n-1}{2}\right)}^{p}, n-1}(\tilde{\omega}) \mid \mathcal{F}_{t \gamma^{-1}, n-1}\right]
$$

where we used the equality

$$
\mathcal{Y}_{t \gamma^{-1}, n-2}=\mathcal{Y}_{t \delta_{\left(\frac{n-1}{2}\right)^{p}}^{\gamma^{-1}, n-2}}
$$

We obtained thus the formulas (55)-(57) and (61)-(63), recursive in $n$, which allow us to express $E_{t, n}$ and $F_{t, n}$ in terms of $E_{t, n-1}$ and $F_{t, n-1}$ (which are, by definition, evaluated at words of shorter length).

\subsection{Barycenters and Reflection Coefficients}

As in the previous section, the Levinson recursions on the prediction errors can be replaced by scalar recursions involving only the barycenters $e_{t, n}$ and $f_{t, n}$ of the forward and backward prediction errors. We define

$$
\begin{aligned}
e_{t, n} & \triangleq q^{\left\lfloor\frac{n-1}{2}\right\rfloor} \sum_{|\omega|<n, \omega \asymp 0} E_{t, n}(\omega) \\
f_{t, n} & \triangleq q^{\left\lfloor\frac{n}{2}\right\rfloor} \sum_{|\omega|=n} F_{t, n}(\omega) .
\end{aligned}
$$


These barycenters are closely related to the set of barycenters defined in Section 3, in particular we have for the barycenter $f_{t, n}$ of the backward prediction error the equality

$$
f_{t, n}=e_{n}\left(t \gamma^{-\left\lfloor\frac{n-1}{2}\right\rfloor-1}, t \gamma^{-\left\lfloor\frac{n-1}{2}\right\rfloor}\right),
$$

while the barycenter $e_{t, n}$ of the forward prediction error satisfy the following equalities

for $n$ even:

$$
\frac{1}{q}\left(e_{t, n}+e_{t \delta_{\left(\frac{n}{2}\right)}}+\cdots+e_{t \delta_{\left(\frac{\pi}{2}\right)}^{q-1}}\right)=e_{n}\left(t \gamma^{-\frac{n}{2}}, t \gamma^{-\frac{n}{2}-1}\right)
$$

for $n$ odd:

$$
e_{t, n}=e_{n}\left(t \gamma^{-\frac{n-1}{2}}, t \gamma^{-\frac{n+1}{2}}\right)
$$

The barycentric Levinson recursions can be directly obtained from the oriented Levinson formulas. Unfortunately we have to use this approach to prove the first equation of the following theorem. 'This is due to the fact that, according to equation (67), $e_{t, n}$ for $n$ even does not represent one of the barycenters $e_{n}(.,$.$) , introduced in section 3$. The other equations, however, will be derived from the corresponding formulas in the unoriented case.

Theorem 4 (Recursions for the barycenters) We obtain the following formulas: $n$ even:

$$
\begin{aligned}
e_{t, n} & =e_{t, n-1}-k_{n} f_{t \gamma^{-1}, n-1} \\
f_{t, n} & =\frac{1}{q}\left(f_{t \gamma-1, n-1}+e_{t \delta_{\left(\frac{n}{2}\right), n-1}}+\cdots+e_{t \delta_{\left(\frac{n}{2}\right)}^{q-1}, n-1}\right)-k_{n} e_{t, n-1},
\end{aligned}
$$

where

$$
\begin{aligned}
k_{n} & =\operatorname{cor}\left(e_{t \delta_{\left(\frac{n}{2}\right), n-1}^{r}}, e_{t \delta_{\left(\frac{n}{2}\right)}^{p}, n-1}\right) \\
& =\operatorname{cor}\left(f_{t \gamma^{-1}, n-1}, e_{t \delta_{\left(\frac{n}{2}\right)}^{p}, n-1}\right)
\end{aligned}
$$

and $r$ and $p$ are such that $0 \leq r \leq q-1,0 \leq p \leq q-1$ and $r \neq p$.

$n$ odd:

$$
\begin{aligned}
& e_{t, n}=\frac{1}{q}\left(e_{t, n-1}+\cdots+e_{t \delta_{\left(\frac{n-1}{2}\right)}^{q-1}, n-1}\right)-k_{n} f_{t \gamma^{-1}, n-1} \\
& f_{t, n}=f_{t \gamma^{-1}, n-1}-\frac{1}{q} k_{n}\left(e_{t, n-1}+\ldots+e_{t \delta_{\left(\frac{n-1}{2}\right)}^{q-1}, n-1}\right),
\end{aligned}
$$

where

$$
k_{n}=\operatorname{cor}\left(\frac{1}{q}\left(e_{t, n-1}+\ldots+e_{t \delta_{\left(\frac{n-1}{2}\right)}^{q-1}, n-1}\right), f_{t \gamma^{-1}, n-1}\right)
$$

Proof: As we mentioned before, we have to show equation (69) explicitly. Using Lemma 7 (cf. Appendix A) we can rewrite equation (57) with the conditional expectation based on the scalar $f_{t \gamma^{-1}, n-1}$. By taking the barycenters of the involved vectors we obtain

$$
e_{t, n}=e_{t, n-1}-\mathbf{E}\left[e_{t, n-1} \mid f_{t \gamma-1, n-1}\right]
$$


i.e. it remains to show, that $\operatorname{cor}\left(e_{t, n-1}, f_{t \gamma^{-1}, n-1}\right)=k_{n}$, which is trivial, using equations (66),(68) and the definition (20) of $k_{n}$. All other formulas can be derived from the corresponding equations in section 3. We will examine here only the case of the backward prediction error for $n$ even (the proofs of the other cases follow essentially the same outline and will be left to the reader). We recall the formula

$$
f_{t, n}=e_{n}\left(t \gamma^{-\frac{n}{2}}, t \gamma^{-\frac{n}{2}+1}\right)
$$

Equation (19) applied at the odd integer $n-1$ yields

$$
e_{n}\left(t \gamma^{-\frac{n}{2}}, t \gamma^{-\frac{n}{2}+1}\right)=\frac{1}{q} \sum_{i=1}^{i=q} e_{n-1}\left(t_{i}, t \gamma^{-\frac{n}{2}}\right)-k_{n} e_{n-1}\left(t \gamma^{-\frac{n}{2}+1}, t \gamma^{-\frac{n}{2}}\right),
$$

the set of all neighboring nodes $t_{i}$ of $t \gamma^{-\frac{n}{2}}$, except the node $t \gamma^{-\frac{n}{2}+1}$, can be rewritten

$$
\left\{t_{i}, i=1, \ldots, q\right\}=\left\{t \gamma^{-\frac{n}{2}-1}, t \gamma^{-\frac{n}{2}+1} \delta_{(1)}^{p}, p=1, \ldots, q-1\right\} .
$$

Using (68) and (66), we get easily

$$
\begin{aligned}
f_{t \gamma^{-1}, n-1} & =e_{n-1}\left(\left(t \gamma^{-1}\right) \gamma^{-\frac{n}{2}},\left(t \gamma^{-1}\right) \gamma^{-\frac{n}{2}+1}\right) \\
& =e_{n-1}\left(t \gamma^{-\frac{n}{2}-1}, t \gamma^{-\frac{n}{2}}\right)
\end{aligned}
$$

and

$$
\begin{aligned}
e_{t \delta_{\left(\frac{n}{2}\right)}^{p}, n-1} & =e_{n-1}\left(t \delta_{\left(\frac{n}{2}\right)}^{p} \gamma^{-\frac{n-2}{2}}, t \delta_{\left(\frac{n}{2}\right)}^{p} \gamma^{-\frac{n-2}{2}-1}\right) \\
& =e_{n-1}\left(t \gamma^{-\frac{n}{2}+1} \delta_{(1)}^{p}, t \gamma^{-\frac{n}{2}}\right)
\end{aligned}
$$

Therefore, the unoriented recursion formula (76) provides us exactly with the sought-for equation. $\square$ The results on the variances of the prediction errors become here

Corollary 2 For $n$ even :

$$
\begin{aligned}
\sigma_{e, n}^{2} & \triangleq \mathbb{E}\left(\left(e_{t, n}^{2}\right)\right. \\
& =\left(1-k_{n}^{2}\right) \sigma_{n-1}^{2} \\
\sigma_{f, n}^{2} & \triangleq \mathbb{E}\left(\left(f_{t, n}^{2}\right)\right. \\
& =\left(\frac{1}{q}+\left(1-\frac{1}{q}\right) k_{n}-k_{n}^{2}\right) \sigma_{n-1}^{2}
\end{aligned}
$$

where $k_{n}$ must satisfy

$$
-\frac{1}{q} \leq k_{n} \leq 1
$$

For $n$ odd :

$$
\begin{aligned}
\sigma_{n}^{2} & \triangleq \sigma_{e, n}^{2}=\sigma_{f, n}^{2} \\
& =\left(1-k_{n}^{2}\right) \sigma_{f, n-1}^{2}
\end{aligned}
$$

where $k_{n}$ must satisfy

$$
-1 \leq k_{n} \leq 1
$$

Proof: The proof of the corollary is essentially the same as the one of the corresponding corollary in the previous section, we leave it to the reader. 


\subsection{Schur Recursions and Computations of the Reflection Coefficients}

We establish here "oriented" Schur recursions using formal power series not in $z$, but in the shifts $\omega \in \mathcal{L}$ on the tree. Let $S$ be a formal power series of the form :

$$
S=\sum_{\omega \in \mathcal{L}} s_{\omega} \omega
$$

We will use the following notations:

$$
\gamma[S] \triangleq \sum_{\omega \in \mathcal{L}} s_{\omega \gamma}-1 \omega
$$

and

$$
\delta_{(k)}^{p}[S] \triangleq \sum_{\omega \in \mathcal{L}} s_{\omega \delta_{(k)}^{p}} \omega
$$

(where $1 \leq p \leq q-1$ ), and we note $S(0)$ the coefficient associated to $\omega=0$. We define the following series:

$$
\begin{aligned}
P_{n} & \triangleq \sum_{\omega \in \mathcal{L}} \mathbb{E}\left(Y_{t} e_{t \omega, n}\right) \cdot \omega \\
Q_{n} & \triangleq \sum_{\omega \in \mathcal{L}} \mathbb{E}\left(Y_{t} f_{t \omega, n}\right) \cdot \omega
\end{aligned}
$$

where $n \geq 0$, which yields for $n=0$ the initial condition of our Schur-recursions:

$$
P_{0}=Q_{0}=\sum_{\omega \in \mathcal{L}} r_{|\omega|} \cdot \omega
$$

where $r_{n}$ is the covariance function of the process $\left\{Y_{t}\right\}_{t \in \mathcal{T}}$.

Theorem 5 (Schur recursions) We obtain the following recursion formulas:

$n$ even:

$$
\begin{aligned}
P_{n} & =P_{n-1}-k_{n} \gamma\left[Q_{n-1}\right] \\
Q_{n} & =\frac{1}{q}\left(\gamma\left[Q_{n-1}\right]+\delta_{\left(\frac{n}{2}\right)}\left[P_{n-1}\right]+\ldots+\delta_{\left(\frac{n}{2}\right)}^{q-1}\left[P_{n-1}\right]\right)-k_{n} P_{n-1}
\end{aligned}
$$

where

$$
k_{n}=\frac{\gamma\left[Q_{n-1}\right](0)+\delta_{\left(\frac{n}{2}\right)}\left[P_{n-1}\right](0)+\ldots+\delta_{\left(\frac{n}{2}\right)}\left[P_{n-1}\right](0)}{q P_{n-1}(0)}
$$

$n$ odd:

$$
\begin{aligned}
P_{n} & =\frac{1}{q}\left(P_{n-1}+\delta_{\left(\frac{n-1}{2}\right)}\left[P_{n-1}\right]+\ldots+\delta_{\left(\frac{n-1}{2}\right)}^{q-1}\left[P_{n-1}\right]\right)-k_{n} \gamma\left[Q_{n-1}\right] \\
Q_{n} & =\gamma\left[Q_{n-1}\right]-\frac{1}{q}\left(P_{n-1}+\delta_{\left(\frac{n-1}{2}\right)}\left[P_{n-1}\right]+\ldots+\delta_{\left(\frac{n-1}{2}\right)}^{q-1}\left[P_{n-1}\right]\right)
\end{aligned}
$$

where

$$
k_{n}=\frac{q \gamma\left[Q_{n-1}\right](0)}{\left(P_{n-1}(0)+\delta_{\left(\frac{n-1}{2}\right)}\left[P_{n-1}\right](0)+\ldots+\delta_{\left(\frac{n-1}{2}\right)}^{q-1}\left[P_{n-1}\right](0)\right)}
$$

Proof : Premultiplying the recursions (69), (70) and (73), (74) by $Y_{t}$ and taking expectations, we obtain these recursions. The computation of the reflection coefficients $k_{n}$ is derived from the fact that $Q_{n}(0)=0$. 


\section{Conclusion}

In this paper we developed a characterization of isotropic processes on a homogeneous tree of order $q$ via a reflection coefficient sequence. The relation between the covariance sequence and the reflection coefficient sequence of a process is derived by generalized Levinson and Schur recursions respectively. Although these results are easily obtained using an isometric (i.e. non-oriented) approach, the Levinson and Schur recursions are also given from the pyramidal point of view. They turn out to be useful in this form for the implementation of generating and whitening filters $[1,2]$.

A central issue in this theory is that of fitting isotropic autoregressive models from data. The "data" we consider are just ordinary signals (for the case $q=2$ ) or images (for $q=4$ ), i.e., we want to fit models of processes on the tree from observations taken at one single horocycle. This is a much more difficult problem than its counterpart for ordinary time series, since only the evenindexed part of the covariance sequences can be estimated from data at a single horocycle. This problem and other related issues will be reported in $[4,5]$. 


\section{Appendix}

\section{A Proofs for the isotropic point of view}

\section{A.1 Some general results on isotropic processes}

Let $Y$ be an isotropic process indexed by the nodes of a homogeneous tree $\mathcal{T}$ of order $q$. Let $\mathcal{T}^{\prime}$ be a subset of $\mathcal{T}$. Then we denote

$$
\mathcal{Y}\left(\mathcal{T}^{\prime}\right) \triangleq \operatorname{span}\left\{Y_{t}, t \in \mathcal{T}^{\prime}\right\}
$$

Given a pair $\left(t, t^{\prime}\right)$ of neighboring nodes (i.e. $d\left(t, t^{\prime}\right)=1$ ), we define

$$
\Omega_{t, n} \triangleq\left\{\omega \in \mathcal{T} \mid d(\omega, t)<d\left(\omega, t^{\prime}\right), d(\omega, t) \leq n-1\right\}
$$

to be a sub-tree of $\mathcal{T}$. We denote

$$
U_{k, t} \triangleq\left\{\omega \in \Omega_{t, n} \mid d(\omega, t)=k \leq n\right\}
$$

a horocycle of $\Omega_{t, n}$. For $U$ being a horocycle we define the barycenter

$$
\bar{Y}_{u} \triangleq \frac{1}{\#} \sum_{\omega \in U} Y_{\omega}
$$

where \# denotes the number of elements in $U$. With an argument of isometries one can show the following result:

Lemma 2 Let $U, U^{\prime}$ be horocycles of a sub-tree $\Omega_{t, n}$. Let $Y_{u}, Y_{u^{\prime}}$ be column-vectors which elements are indexed by the nodes of $U, U^{\prime}$ respectively, i.e. $Y_{u}=\left\{Y_{t}\right\}_{t \in U}$. Then the covariance-matrix $\Sigma_{u}=\mathbb{E}\left(Y_{u} Y_{u}^{T}\right)$ has the eigenvector $\mathbb{1}=(1, \ldots, 1)^{T}$, and $\Sigma_{u, u^{\prime}}=\mathbb{E}\left(Y_{u} Y_{u^{\prime}}^{T}\right)$ has also the eigenvector $\mathbb{1}$, in that sense that

$$
\Sigma_{u, u^{\prime}} \mathbb{1}=c \mathbb{1} \text { and } \mathbb{1}^{T} \Sigma_{u, u^{\prime}}=c^{\prime} \mathbb{1}^{T} .
$$

where the vectors are of appropriate dimensions.

Lemma 3 Let $U, U^{\prime}$ be horocycles of a sub-tree $\Omega$, let $\bar{Y}_{u}, \bar{Y}_{u^{\prime}}$ be the associated barycenters. Then we have

$$
\mathbb{E}\left(\bar{Y}_{u} \mid \mathcal{Y}\left(U^{\prime}\right)\right)=\mathbb{E}\left(\bar{Y}_{u} \mid \bar{Y}_{u^{\prime}}\right)
$$

and, for $t, t^{\prime} \in U^{\prime}$,

$$
\mathbb{E}\left(Y_{t} \mid \bar{Y}_{u}\right)=\mathbb{E}\left(Y_{t^{\prime}} \mid \bar{Y}_{u}\right)
$$

Proof: Using the fact that $\mathbb{1}$ is a eigenvector of $\Sigma_{U}, \Sigma_{u, u^{\prime}}$ the result follows immediately. $\square$

Lemma 4 Let $\Omega_{t, \infty}, \Omega_{s, \infty}$ be two semi-infinite disjoint sub-trees, and let $U \subset \Omega_{t, \infty}$ and $V \subset \Omega_{s, \infty}$ be horocycles of $\Omega_{t, \infty}, \Omega_{s, \infty}$ respectively. Let $v_{1}, v_{2} \in V$, then we have

$$
\begin{aligned}
\mathbb{E}\left(Y_{v_{1}} \mid \mathcal{Y}(U)\right) & =\mathbf{E}\left(Y_{v_{1}} \mid \bar{Y}_{u}\right) \\
& =\mathbb{E}\left(Y_{v_{2}} \mid \bar{Y}_{u}\right)
\end{aligned}
$$


Proof: By using the orthogonal sum $\mathcal{Y}(U)=\left[\bar{Y}_{u}\right] \oplus \mathcal{Y}_{u}^{-}$we find that

$$
\mathbb{E}\left(Y_{s} \mid \mathcal{Y}_{u}^{-}\right)=0 .
$$

(96) is an immediate result of the isotropy of the process $Y$.

Let now $\Omega_{1} \triangleq \Omega_{t, n} \subset \Omega_{t, \infty}$ and $\Omega_{2} \triangleq \Omega_{s, m} \subset \Omega_{s, \infty}$ be finite sub-trees of $\mathcal{T}$. Let $\Omega_{3} \subset \mathcal{T} \backslash$ $\left\{\Omega_{t, \infty}, \Omega_{s, \infty}\right\}$ be an arbitrary set of nodes, and note $\tilde{\Omega}=\Omega_{1} \cup \Omega_{2} \cup \Omega_{3}$. Let $\mathcal{U}, \mathcal{V}$ be horocycles of $\Omega_{t, \infty}, \Omega_{s, \infty}$ such that $\Omega_{t, n} \cup \mathcal{U}=\Omega_{t, n+1}$ and $\Omega_{s, m} \cup \mathcal{V}=\Omega_{s, m+1}$.

Lemma 5 Let $\omega \in \Omega_{2}, \Omega_{1}=\bigcup_{i} U_{i}$ where $U_{i}$ are the horocycles of $\Omega_{1}$, then we have

$$
\mathbb{E}\left(Y_{\omega} \mid \mathcal{Y}\left(\Omega_{1}\right)\right)=\mathbb{E}\left(Y_{\omega} \mid\left[\bar{Y}_{u_{i}}\right]\right)
$$

and

$$
\mathbb{E}\left(\bar{Y}_{u,} \mid \mathcal{Y}\left(\Omega_{2} \backslash U_{j}\right)\right)=\mathbb{E}\left(\bar{Y}_{u}, \mid\left[\bar{Y}_{u_{i}}, i \neq j\right]\right)
$$

Proof: Equation (98) can easily be shown by using (93), and (97) follows by using (98) and (95).

We define the general prediction error

$$
F_{t} \triangleq Y_{t}-\mathbb{E}\left(Y_{t} \mid \mathcal{Y}(\tilde{\Omega})\right)
$$

and $\mathcal{F}\left(\mathcal{T}^{\prime}\right)=\operatorname{span}\left\{F(t), t \in \mathcal{T}^{\prime}\right\}$ where $\mathcal{T}^{\prime} \subset \mathcal{T}$ is a set of nodes. We denote $\bar{F}$ the associated barycenter: $\bar{F}_{v}=\frac{1}{\#} \sum_{v \in \mathcal{V}} F_{v}$.

Lemma 6 Let $u, u^{\prime} \in \mathcal{U}, v, v^{\prime} \in \mathcal{V}$, then we have

$$
\mathbb{E}\left(F_{u} \mid \mathcal{F}(\mathcal{V})\right)=\mathbb{E}\left(F_{u^{\prime}} \mid \mathcal{F}(\mathcal{V})\right)
$$

and

$$
\mathbb{E}\left(F_{u} F_{v}\right)=\mathbb{E}\left(F_{u^{\prime}} F_{v^{\prime}}\right)=\mathbb{E}\left(Y_{u} F_{v}\right)
$$

Proof: By definition we have $\mathbb{E}\left(F_{u} F_{v}\right)=\mathbb{E}\left(Y_{u} F_{v}\right)=\mathbb{E}\left(F_{u} Y_{v}\right)$. One can show that $F_{v}$ is a linear combination of $\bar{Y}_{U_{v}}$ and $Y_{s}, s \in \mathcal{V} \cup \tilde{\Omega} \backslash \Omega_{1}$ (by using (97)). Using the isotropy and (94) we find (101) which leads us immediately to (100).

By using (101) recursively it can be shown that the covariance-matrix $\Sigma_{F_{u}}=\mathbb{E}\left(F_{u} F_{u}^{T}\right)$, where $F_{u}=\left\{F_{t}\right\}_{t \in U}$ has $\mathbb{1}$ as a eigenvector, and with the same proof as in (93), (94) we obtain the following lemma:

Lemma 7 We have

$$
\begin{aligned}
\mathbb{E}\left(F_{u} \mid \mathcal{F}_{v}\right) & =\mathbb{E}\left(F_{u} \mid \bar{F}_{v}\right) \\
& =\mathbb{E}\left(F_{u^{\prime}} \mid \bar{F}_{v}\right)
\end{aligned}
$$

This lemma allowed us to replace in equations (13) and (14) the conditional expectations based on the prediction error spaces $\mathcal{E}_{n}(t, s)$ by the conditional expectations based on the scalars $e_{n}(t, s)$. 


\section{A.2 Some results on covariances}

Up to now, most random variables have been defined by giving one or more nodes of the tree as determining reference nodes. In this section we will show that certain covariances yield the same results if only the determining nodes of the involved random variables have the same "relative position" to each other. These results justify the introduction of the reflection coefficients $k_{i}$ and the "one-dimensional" Schur-recursions.

Lem ma 8 Let $\left(s_{1}, s_{2}\right)$ be an oriented branch of the tree $\mathcal{T}$. Then, for fixed $n$, the expression

$$
\mathbb{E}\left(Y_{t} e_{n}\left(s_{1}, s_{2}\right)\right)
$$

depends only on the distance $d\left(t, s_{1}\right)$ and the relative orientation of the branch $\left(s_{1}, s_{2}\right)$ with respect to the node $t$, i.e. on the sign of $d\left(s_{1}, t\right)-d\left(s_{2}, t\right)$.

Proof: Let $s_{1}, s_{2}$ be fixed. The prediction error $E_{n, s_{1}, s_{2}}(v)$ is defined for $v \in V_{\left\lfloor\frac{n}{2}\right\rfloor}\left(s_{1}, s_{2}\right)$ to be the residual of the projection of $Y_{v}$ on the space spanned by $Y_{u}, u \in B_{n-1}\left(s_{2}, s_{1}\right)$. The set of nodes $B_{n-1}\left(s_{2}, s_{1}\right) \cup V_{\left\lfloor\frac{n}{2}\right\rfloor}\left(s_{1}, s_{2}\right)$ represents the union of two finite, disjoint sub-trees, $\Omega_{1}$ with root-node $s_{1}$ and $\Omega_{2}$ with root-node $s_{2}$. $V_{\left\lfloor\frac{n}{2}\right\rfloor}\left(s_{1}, s_{2}\right)$ constitutes now a horocycle of $\Omega_{1}$. We have

$$
\begin{aligned}
e_{n}\left(s_{1}, s_{2}\right)= & \frac{1}{\#} \sum_{v \in V_{\left\lfloor\frac{n}{2}\right\rfloor}\left(s_{1}, s_{2}\right)}\left(Y_{v}-\mathbb{E}\left(Y_{v} \mid \mathcal{Y}\left(B_{n-1}\left(s_{2}, s_{1}\right)\right)\right)\right) \\
= & \bar{Y}_{v}-\mathbb{E}\left(\bar{Y}_{v} \mid \mathcal{Y}\left(B_{n-1}\left(s_{2}, s_{1}\right)\right)\right) \\
= & \bar{Y}_{v}-\mathbb{E}\left(\bar{Y}_{v} \mid \mathcal{Y}\left(\Omega_{2}\right)\right)-\mathbb{E}\left(\bar{Y}_{v} \mid \mathcal{Y}\left(\Omega_{1} \backslash V_{\left\lfloor\frac{n}{2}\right\rfloor}\left(s_{1}, s_{2}\right)\right)\right) \\
& +\mathbb{E}\left(\mathbb{E}\left(\bar{Y}_{v} \mid \mathcal{Y}\left(\Omega_{2}\right)\right) \mid \mathcal{Y}\left(\Omega_{1} \backslash V_{\left\lfloor\frac{n}{2}\right\rfloor}\left(s_{1}, s_{2}\right)\right)\right)
\end{aligned}
$$

which is a linear combination of the barycenters of $Y$ with respect to the horocycles of $\Omega_{1}, \Omega_{2}$. This is easily to be seen by using (97) and (98). The equivalence classes of nodes with respect to the branch $\left(s_{1}, s_{2}\right)$, i.e. the set of nodes lying at the same distance to $s_{1}$ and "on the same side" of the branch $\left(s_{1}, s_{2}\right)$ constitute horocycles of the semi-infinite trees $\tilde{\Omega}\left(s_{1}\right), \tilde{\Omega}\left(s_{2}\right)$ (such that $\left.\tilde{\Omega}\left(s_{1}\right) \cap \tilde{\Omega}\left(s_{2}\right)=\emptyset, s_{1} \in \tilde{\Omega}\left(s_{1}\right), s_{2} \in \tilde{\Omega}\left(s_{2}\right)\right)$. With (94),(96) we find now that

$$
\mathbb{E}\left(Y_{t} e_{n}\left(s_{1}, s_{2}\right)\right)
$$

is a constant for all $t$ in one equivalence class. Note now the fact that for a branch $\left(\tilde{s}_{1}, \tilde{s}_{2}\right)$ each isometry $\Phi$ with $\Phi\left(\left(s_{1}, s_{2}\right)\right)=\left(\tilde{s}_{1}, \tilde{s}_{2}\right)$ satisfies $\Phi(\tilde{\Omega}(s))=.\tilde{\Omega}\left(\tilde{s}_{\text {s. }}\right)$, and in particular the image of each horocycle of $\tilde{\Omega}(s$.) is the corresponding horocycle of $\tilde{\Omega}(\tilde{s}$.). This yields the result.

Lemma 9 Let $\left(s_{1}, s_{2}\right),\left(t_{1}, t_{2}\right)$ be branches of the tree $\mathcal{T}$. Let $\Omega_{s_{1}, \infty}, \Omega_{t_{1}, \infty}$ be semi-infinite subtrees such that $s_{2} \notin \Omega_{s_{1}, \infty}, t_{2} \notin \Omega_{t_{1}, \infty}$. Let $U_{i, s_{1}}, U_{j, t_{1}}, i, j \in \mathbb{N}$ be the horocycles of $\Omega_{s_{1}, \infty}$ and $\Omega_{t_{1}, \infty}$ respectively (where the $U_{i, .}$ are numbered such that $U_{i, .}$ has $2^{i}$ elements), we denote then by $\bar{Y}_{i, s_{1}}, \bar{Y}_{j, \ell_{1}}$ the associated barycenters of the isotropic process $Y$. Now the covariance

$$
\mathbb{E}\left(\bar{Y}_{m, s_{1}} \bar{Y}_{n, t_{1}}\right)
$$


depends only on $m, n$, the distance $d\left(s_{1}, t_{1}\right)$ and on the relative orientation of the two branches $\left(s_{1}, s_{2}\right)$ and $\left(t_{1}, t_{2}\right)$, i.e. on $\frac{d\left(s_{1}, t_{1}\right)-d\left(s_{2}, t_{2}\right)}{2}$ which can take the following values

$$
\frac{d\left(s_{1}, t_{1}\right)-d\left(s_{2}, t_{2}\right)}{2}=\left\{\begin{array}{cl}
1 & , \text { if the branches are opposed } \\
0 & , \text { if the branches have the same orientation } \\
-1 & , \text { if the branches are divergent }
\end{array}\right.
$$

Proof: For given branches $\left(s_{1}, s_{2}\right),\left(t_{1}, t_{2}\right),\left(\tilde{s}_{1}, \tilde{s}_{2}\right),\left(\tilde{t}_{1}, \tilde{t}_{2}\right)$ with $d\left(s_{1}, t_{1}\right)=d\left(\tilde{s}_{1}, \tilde{t}_{1}\right)$ and $\frac{d\left(s_{1}, t_{1}\right)-d\left(s_{2}, t_{2}\right)}{2}=\frac{d\left(\tilde{s}_{1}, \tilde{t}_{1}\right)-d\left(\tilde{s}_{2}, \tilde{t}_{2}\right)}{2}$ there exists an isometry $\Phi$ such that

$$
\Phi\left(\left[\left(s_{1}, s_{2}\right),\left(t_{1}, t_{2}\right)\right]\right)=\left[\left(\tilde{s}_{1}, \tilde{s}_{2}\right),\left(\tilde{t}_{1}, \tilde{t}_{2}\right)\right]
$$

and in particular we have

$$
\Phi\left(\Omega_{s_{1}, \infty}\right)=\Omega_{\tilde{s}_{1}, \infty} \text { and } \Phi\left(U_{i, s_{1}}\right)=U_{i, \bar{s}_{1}},
$$

the analogous equalities are satisfied for $\Omega_{t_{1}, \infty}$ and $U_{i, t_{1}}$ which yields immediately the result.

With equation (105) we saw that $e_{n}\left(s_{1}, s_{2}\right)$ can be rewritten as a linear combination of the barycenters of the process $Y$ with respect to the horocycles of certain finite subtrees $\Omega_{1}, \Omega_{2}$. As the coefficients in this linear combination do not depend on the choice of the branch $\left(s_{1}, s_{2}\right)$, we can apply Lemma 9 to $\mathbb{E}\left(e_{n}\left(t_{1}, t_{2}\right) e_{n}\left(s_{1}, s_{2}\right)\right)$ and we obtain easily the following corollary

Corollary 3 The covariance $\mathbb{E}\left(e_{n}\left(t_{1}, t_{2}\right) e_{n}\left(s_{1}, s_{2}\right)\right.$ ) (for fixed $n$ ) depends only on the distance $d\left(s_{1}, t_{1}\right)$ and on the relative orientation of the oriented branches $\left(s_{1}, s_{2}\right)$ and $\left(t_{1}, t_{2}\right)$.

This corollary allowed us to introduce in Section 3.2 the reflection coefficients $k_{i}$.

\section{References}

[1] M. Basseville, A. Benveniste, A.S. Willsky, "Multi-scale Autoregressive Processes, Part I: Schur-Levinson Parametrizations," to appear in IEEE Transactions on ASSP, August 1992

[2] M. Basseville, A. Benveniste, A.S. Willsky, "Multi-scale Autoregressive Processes, Part II: Lattice Structures for Whitening and Modeling," to appear in IEEE Transactions on ASSP, August 1992

[3] M. Basseville et al., "Modeling and Estimation of Multiresolution Stochastic Processes," to appear in IEEE Trans. Inf. Theory, special issue on wavelets, 1992

[4] B. Claus, A. Benveniste, "On the Covariance-sequence of AR-processes - An Interpolation Problem and its Extension to Multiscale AR-Processes," in preparation

[5] B. Claus , "Identification of AR-Processes on Dyadic Trees," in preparation

[6] R. Coifman, M.V. Wickerhauser, "Entropy-based Algorithms for Best Basis Selection," to appear in IEEE Trans. Inf. Theory, special issue on wavelets, 1992 
[7] R.E. Crochiere, L.R. Rabiner, Multirate Digital Signal Processing, Englewood Cliffs, NJ, Prentice Hall, 1983

[8] I. DAubechies, "Orthonormal bases of compactly supported wavelets," Communications on Pure and Applied Math., vol.91, pp. 909-996, 1988 


\section{LISTE DES DERNIERES PUBLICATIONS INTERNES \\ PARUES A L'IRISA}

PI 648 SET-THEORETIC GRAPH REWRITING Jean-Claude RAOULT, Frédéric VOISIN Mars 1992,18 pages.

PI 649 UNE STRUCTURE D'INFORMATION POUR LES ALGORITHMLS D'EXCLUSION MUTUELLE FONDES SUR UNE ARBORESCENCE

Jean-Michel HELARY, Achour MOSTEFAOUI, Michel RAYNAL

Mars 1992, 18 pages.

PI 650 BLOCK-ARNOLDI AND DAVIDSON METHODS FOR UNSYMMETRIC LARGE EIGENVALUE PROBLEMS

Miloud SADKANE

Avril 1992, 24 pages.

PI 651 COMPILING SEQUENTIAL PROGRAMS FOR DISTRIBUTED MEMORY PARALLEL COMPUTERS WITH PANDORE II

Françoise ANDRE, Olivier CHERON, Jean-Louis PAZAT

Avril 1992,18 pages.

PI 652 CHARACTERIZING THE BEHAVIOR OF SPARSE ALGORITHMS ON CACHES Olivier TEMAM, William JALBY

Avril 1992, 20 pages.

PI 653 MADMACS : UN OUTIL DE PLACEMENT ET ROUTAGE POUR LE DESSIN DE MASQUES DE RESEAUX REGULIERS

Eric GAUTRIN, Laurent PERRAUDEAU, Oumarou SIE

Avril 1992, 16 pages.

PI 654 SYSTEN ES D'EQUATIONS RECURRENTES

Patrice QUINTON

Avril 1992, 20 pages.

PI 655 DIFFUSION ON SCALABLE HONEYCOMB NETWORKS

Dominique DESERABLE

Avril 1992, 24 pages.

PI 656 CAUSALITY ORIENTED SHARED MEMORY FOR DISTRIBUTED SYSTEMS

Michel RAYNAL, Masaaki MIZUNO, Mitch NEILSEN

Avril 1992, 8 pages.

PI 657 ALGORITHMES PARALLELES POUR L'ANALYSE D'IMAGE PAR CHAMPS MARKOVIENS

Etienne MEMIN, Fabrice HEITZ

Mai 1992, 74 pages.

PI 658 MULTISCALE SIGNAL PROCESSING : ISOTROPIC RANDOM FIELDS ON HOMOGENEOUS TREES

Bernhard CLAUS, Ghislaine CHARTIER

Mai 1992, 28 pages.

PI 659 ON THE COVARIANCE-SEQUENCE OF AR-PROCESSES. AN INTERPOLATION PROBLEM AND ITS EXTENSION TO MULTISCALE AR-PROCESSES Bernhard CLAUS, Albert BENVENIST'E

Mai 1992, 36 pages.

PI 660 EXCEPTION HANDLING IN COMMUNICATING SEQUENTIAL PROCESSES DESIGN, VERIFICATION AND IMPLEMENTATION

Jcan-Pierre BANATRE, Valérie ISSARNY.

Mai 1992, 38 pages. 


\section{ISSN 0249-6399}

